\title{
СОВРЕМЕННАЯ ДИАГНОСТИКА И ЛЕЧЕНИЕ ГИПЕРПЛАСТИЧЕСКИХ ПРОЦЕССОВ ЭНДОМЕТРИЯ
}

$\mathrm{B}^{\mathrm{s}}$ ысокий риск малигнизации гиперпластических процессов эндометрия, а также повышение частоты гиперплазии и рака эндометрия за последнее десятилетие во многих странах мира, в т.ч. и в Украине, обосновывают актуальность данной проблемы. Сегодня распространенность заболеваемости раком эндометрия занимает третье место среди злокачественных опухолей у женщин и, согласно долгосрочному прогнозу ВОЗ, уже через несколько лет в структуре женской онкологической заболеваемости будет на первом месте в большинстве развитых стран [5].

По данным зарубежных исследователей, половина случаев развития рака эндометрия в пери- и постменопаузе является результатом малигнизации гиперпластических процессов, причем частота и сроки малигнизации колеблются в широких пределах (0,25-50\%) и в значительной мере зависят от степени выраженности гиперпролиферации в эндометрии $[23,25]$. Именно поэтому своевременная диагностика и адекватная терапия гиперплазии эндометрия (ГЭ) является залогом успешной профрилактики рака эндометрия.

До сих пор дискутабельным остается вопрос относительно трактования разных видов ГЭ как предраковых состояний и соответственно частоты их малигнизации. В связи с этим особого внимания заслуживают вопросы терминологии, используемой для оценки различных видов гиперпролиферативных процессов эндометрия, что чрезвычайно важно для их унифицированной клинической интерпретации.

Согласно распространенной сегодня классифрикации, разработанной и утвержденной ВОЗ (1994), выделяют:

э простую и комплексную неатипические ГЭ; простую и комплексную атипические ГЭ;

заденокарциному (схема 1).

Следует отметить, что в соответствии с данной классификацией ВОЗ (1994), которой до настоящего времени пользуются клиницисты и патологи во всем мире, комплексная неатипическая гиперплазия по старой классификации ВОЗ (1975) соответствует понятию «аденоматоз» и не идентифицируется с понятием «атипия». Основным ее отличием от простой гиперплазии является железистое переполнение, т.е. нарушение баланса между пролиферацией желез и стромы, тогда как для простой гиперплазии характерно увеличение объема эн- дометрия с равномерной пролиферацией как железистого, так и стромального компонентов. Укоренившийся из старой классификации синоним комплексной гиперплазии (аденоматозная ГЭ) до сих пор используется некоторыми гистологами постсоветского пространства и при этом порой расценивается клиницистами как пограничное состояние эндометрия, что влечет за собой часто необоснованную радикализацию лечебных мер $[10,15,16]$.

Согласно упомянутой классификации ВОЗ (1994), диагностическим критерием предраковых состояний эндометрия является наличие цитологической атипии. Признаки клеточной атипии могут отмечаться как на фоне простой, так и на фоне комплексной гиперплазий (схема 1) [10].

В данной классификации ГЭ отдельно не выделен термин «полип эндометрия», ибо он трактуется как результат продуктивного хронического эндометрита, что требует бактериологического обследования и адекватного противовоспалительного лечения. Гормональная терапия в этих случаях определяется особенностями морфофункциональной структуры эндометрия, отражающей те или иные изменения эндокринного гомеостаза [14, 15].

В многочисленных исследованиях последних десятилетий было доказано, что простая и комплексная неатипические ГЭ являются результатом абсолютной или относительной гиперэстрогении, в то время как атипическая ГЭ представляет собой прогрессирующее моноклональное мутационное повреждение с независимым от гормонального воздействия локальным ростом. С учетом этого был предложен термин «эндометриальная интраэпителиальная неоплазия» (Endometrial Intraepithelial Neoplasia, EIN), которую следует рассматривать как предрак (табл. 1) [4, 30]. Гистологическими признаками интраэпителиальной неоплазии является железистое переполнение, т.е. уменьшение объемного соотношения стромы желез, при котором объем железистого компонента составляет более 55\%.

В соответствии с основанной на таком подходе EIN-классификацией выделяют простую и комплексную неатипические ГЭ, интерпретирующиеся как эндометриальная гиперплазия (Endometrial Hyperplasia, EH), являющаяся результатом эстрогенной стимуляции и, следовательно, хорошо поддающаяся гормональной терапии. Простая и комплексная атипические

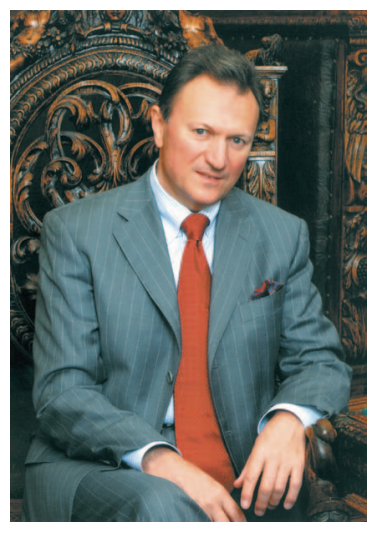

В.Н. ЗАПОРОЖАН

д.мед.н., профессор, академик НАМН Украины, вице-президент НАМН Украины, ректор

Одесского национального медицинского университета, заведующий кафедрой акушерства и гинекологии № 1

Т.Ф. ТАТАРЧУК

д.мед.н., профессор, член-кор. НАМН Украины, заместитель директора по научной работе, заведующая отделением эндокринной гинекологии Института педиатрии, акушерства и гинекологии НАМН Украины

\section{В.Г. ДУБИНИНА}

д.мед.н., профессор кафедры акушерства и гинекологии № 1 , проректор по лечебной работе

Одесского национального медицинского университета, директор Центра реконструктивной $и$ восстановительной медицины, г. Одесса

H.В. КОСЕЙ

д.мед.н., главный научный сотрудник отделения эндокринной гинекологии Института педиатрии, акушерства и гинекологии НАМН Украины 
ТАБЛИЦА 1

\begin{tabular}{|c|c|c|c|}
\hline Номенклатура ВО3 (1994) & Номенклатура EIN & $\begin{array}{c}\text { Функциональная } \\
\text { категория }\end{array}$ & Лечение \\
\hline $\begin{array}{l}\text { Простая неатипическая } \\
\text { гиперплазия }\end{array}$ & \multirow[t]{2}{*}{$\begin{array}{l}\text { Эндометриальная } \\
\text { гиперплазия (ЕН) }\end{array}$} & \multirow{2}{*}{$\begin{array}{l}\text { Эфффект относитель- } \\
\text { ной или абсолютной } \\
\text { гиперэстрогении }\end{array}$} & \multirow[t]{2}{*}{$\begin{array}{c}\text { Гормональная } \\
\text { терапия }\end{array}$} \\
\hline $\begin{array}{l}\text { Сложная (комплексная) } \\
\text { гиперплазия (аденоматоз } \\
\text { без атипии) }\end{array}$ & & & \\
\hline $\begin{array}{l}\text { Простая атипическая } \\
\text { гиперплазия }\end{array}$ & \multirow{2}{*}{$\begin{array}{c}\text { Эндометриальная } \\
\text { интраэпителиальная } \\
\text { неоплазия (EIN) }\end{array}$} & \multirow[t]{2}{*}{ Предрак } & \multirow{2}{*}{$\begin{array}{c}\text { Гормональное или } \\
\text { хирургическое } \\
\text { лечение }\end{array}$} \\
\hline $\begin{array}{l}\text { Сложная (комплексная) } \\
\text { атипическая гиперплазия } \\
\text { (аденоматоз с атипией) }\end{array}$ & & & \\
\hline Аденокарцинома & Аденокарцинома & Рак & Зависит от стадии \\
\hline
\end{tabular}

ТАБЛИЦА 1.

КЛАССИФИКАЦИЯ ГЭ

(GEOGE L. MUTTER, 1999) формы гиперплазии соответствуют понятию эндометриальной интраэпителиальной неоплазии (EIN) и трактуются как предрак, требуя индивидуального подхода и нередко - хирургического лечения [20].

Таким образом, определяющий фактор адекватной диагностики и выбора оптимальной тактики ведения пациенток с ГЭ - интерпретация результатов гистологических заключений. При интерпретации таких заключений чрезвычайно важным является применение унифицированных методов оценки эндометрия клиницистами и гистологами с использованием единой классификации его патологических состояний и определения их функциональной категории [17, 29]. Это возможно при достаточном понимании природы нижеизложенных патологических изменений эндометрия, отражающих функциональные, циркуляторные, метапластические изменения и ятрогенные влияния, т.е. изменения, происходящие в эндометрии под воздействием тех или иных медикаментов (табл. 2).

Функциональные изменения эндометрия, возникающие при ановуляции или эндометриозе, достаточно изучены и известны, тогда как
ГЭ при циркуляторных расстройствах, метапластических процессах и ятрогенных воздействиях заслуживает более пристального внимания [24, 26].

Циркуляторные нарушения проявляются патологическим отеком, лимфатическими кистами и апоплексией эндометрия. Патологический отек является, как правило, следствием нарушения венозного или лимфатического оттока, В т.ч. механической компрессии лимфатических сосудов (неправильное положение матки, субмукозный фриброматозный узел, полип эндометрия), а также результатом гормональной дисфункции с эндогенной гиперэстрогенией либо экзогенного введения гормонов. Лимфратические кисты эндометрия развиваются как результат пересечения или лигирования кровеносных или лимсратических сосудов во время миомэктомии и высокой ампутации матки.

Апоплексия эндометрия возникает при механической обструкции сосудов миомой, нарушении кровообращения при сердечно-сосудистой недостаточности или гипокоагуляционном кровотечении.

В затруднительное положение клинициста ставят патогистологические заключения, указывающие на метапластические изменения. Однако понимание причин, вызывающих эти изменения, помогает в определении дальнейшей тактики ведения пациенток. Муцинозные метаплазии (локализуются в эндоцервиксе) развиваются у постменопаузальных женщин, получавших ЗГТ, гестагены или антиэстрогены. Папиллярные (синцитиальные) метаплазии развиваются также преимущественно у женщин с кровотечениями в постменопаузе или возникают вследствие механического раздражения (например при частых выскабливаниях полости матки). Метаплазии мерцательного эпителия характерны для лиц с пролифреративным

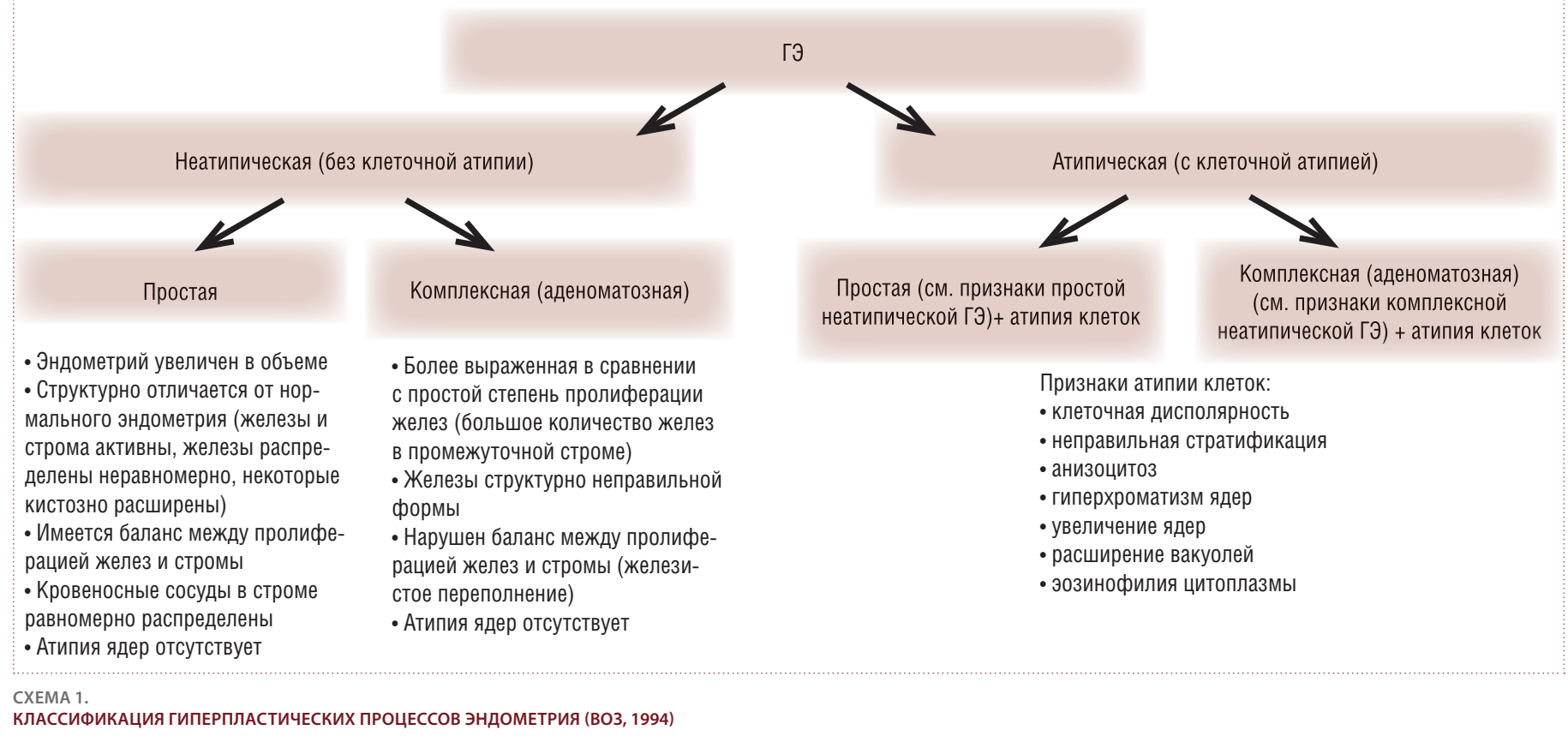


Функциональные нарушения

Ановуляторные нарушения

- Атросрия

- Покоящийся эндометрий

- Десицит пролиферации

- Нарушение пролиферации

Эндометриальная гиперплазия

- Неатипическая (простая и комплексная)

- Атипическая (простая и комплексная)

- Специфические находки в гиперплази-

рованном эндометрии (сквамозная мета-

плазия и метаплазия пенистых клеток)

- Гиперплазия желез и стромы

- Очаговая гиперплазия

- Базальная гиперплазия

- Эндометриальные полипы

Овуляторные нарушения

- Дефицит секреторной фазы

- Нерегулярные месячные (вследствие

длительной регрессии желтого тела и

соответственно медленной секреции

прогестерона)

- Децидуализация эндометрия (дезин-

теграция ретикулярных волокон и

уменьшение релаксина вследствие

персистирующей секреции прогестерона

или терапии гестагенами. После прекращения гестагенной терапии происходит отторжение эндометрия [гормональный кюретаж])

Функциональные эндогенные изменения в течение перименопаузального периода - Секреторная гипертрофия
Изменения в эндометрии, обусловленные циркуляторными нарушениями

\section{Патологический отек}

- нарушение венозного и лимфратическо-

го оттока

- гормональная диссуункция с эндогенной

гиперэстрогенией

- экзогенное введение гормонов

- механическая компрессия лимфати-

ческих сосудов из-за неправильного

положения матки, субмукозного фрибро-

матозного узла или полипа

Лимфатические кисты эндометрия

развиваются вследствие пересечения и лигирования кровеносных и лимфатических сосудов во время гистерэктомии Апоплексия эндометрия

- механическая обструкция сосудов фибромиомой

- нарушение кровообращения при сердечной недостаточности

- агональное или послеоперационное кровотечение

\section{Метапластические изменения}

Эпителиальная метаплазия

- Сквамозные метаплазии и ихти03 (сенильная атрофия)

- Муцинозные метаплазии (эндоцервикальная метаплазия развивается у постменопаузальных женщин, получавших заместительную гормональную терапию

[3ГТ], гестагены или антиэстрогены [тамоксифен])

- Папиллярные (синцитиальные) метаплазии (развиваются у постменопаузальных женщин с кровотечением или вследствие механического раздражения)

- Метаплазия мерцательного эпителия (трубная) - у женщин с пролиферативным и/или гиперпластическим эндометрием во время эстрогенной стимуляции или при ЗГТ

- Редкие формы метаплазии эндометриального железистого эпителия:

- светлоклеточная метаплазия - эозинофильная метаплазия Стромальная метаплазия - Стромальная метаплазия фетального происхождения - Смешанные мезенхимальные опухоли

\section{Ятрогенные изменения}

Комбинированная терапия (комбинированными оральными контрацептивами [KОК] или 3ГТ)

- неполноценная секреция

- неравномерная атрофия

- нерегулярная регенерация, очаго-

вая стромальная гиперплазия

- псевдомеланоз

- очаговая аденоматозная гиперплазия

Терапия гормонами для индукции овуляции

- использование гонадотропинов вызывает неправильные секреторные изменения эндометрия

- применение кломифена приводит к неполноценной секреторной трансформации эндометриальных желез (за счет антиэстрогенного эффекта)

Длительная монотерапия гестагенами или тамоксифеном

- эндоцервикальные метаплазии на фоне покоящегося эндометрия или его атрофиии

- эндоцервикальные атипические гиперплазии и неэндометриальные карциномы

Изменения эндометрия, ассоциированные с модуляторами прогестероновых рецепторов и/или гиперпластическим эндометрием и свидетельствуют о наличии эстрогенной стимуляции. Сквамозная метаплазия и ихтиоз являются следствием сенильной атрофии $[13,26]$.

В связи с широким внедрением в клиническую практику гормональной терапии особое значение приобретают ятрогенные влияния. Ятрогенные изменения эндометрия возникают в результате терапии KOK и ЗГТ длительностью более 6 мес вследствие превалирования эффекта гестагенов. Доминирование гестагенов является причиной развития неполноценной секреции, неравномерной атрофии, нерегулярной регенерации, очаговой стромальной гиперплазии, псевдомеланоза и даже очаговой комплексной гиперплазии. Из вышесказанного следует сделать вывод о том, что ни КОК, ни ЗГТ не являются методом лечения ГЭ.

Длительная монотерапия гестагенами или тамоксифеном, индуцируя атрофию эндометрия, может стимулировать пролиферацию эндоцервикальных желез и клеток резерва, что приводит к развитию эндоцервикальных метаплазий на фоне покоящегося эндометрия или его атрофии.

В аспекте ятрогенного влияния на эндометрий отдельного внимания заслуживают препараты для индукции овуляции. Так, использование гонадотропинов приводит к аномальным секреторным изменениям, а применение кломифена - к неполноценной секреторной трансформации эн- дометриальных желез. Ввиду последнего применение данных препаратов является рациональным только на фоне достаточного эстрогенного обеспечения I фазы менструального цикла [7, 26].

Резюмировать вышесказанное помогают опубликованные G. Dallnebach-Hellweg в 2010 г. данные, где сообщается о типах эндометриальных карцином, развитие которых возможно на фоне применения гормональных препаратов. Хорошо известен фракт, что прием эстрогенов может спровоцировать рак эндометрия, при этом часто отмечается сквамозная диффреренциация. В последние годы обнаружено, что прием гестагенов или тамоксифена может спровоцировать развитие муцинозной, светлоклеточной и серозно-папиллярной метаплазий вплоть до возникновения соответствующих им форм рака: муцинозной, светлоклеточной аденокарцином и серозной карциномы (табл. 3) [26]. Указанные данные обязывают врачей изменить свое привычное отношение к применению гестагенов в лечении гиперпролиферативных процессов эндометрия и осторожно относиться к ним при наличии определенных видов метапластических изменений, отдавая в таких случаях предпочтение использованию других методов лечения, например агонистам гонадотропных рилизинг-гормонов (аГнРГ).

В последние десятилетия появилась новая группа препаратов - модуляторы прогестероновых рецепторов (МПР) (агонисты и антагонисты), 
ТАБЛИЦА 3

\begin{tabular}{r|l|l|}
\multicolumn{2}{|c|}{ Эстрогены } \\
\hline Атипическая гиперплазия $\rightarrow$ & Эндометриоидная аденокарцинома \\
\hline Атипическая гиперплазия со сквамозной & Эндометриоидная аденокарцинома \\
\hline метаплазией $\rightarrow$ & со сквамозной диффреренциацией \\
\hline гестагены/тамоксифен \\
\hline Муцинозная метаплазия $\rightarrow$ & Муцинозная аденокарцинома \\
\hline Светлоклеточная метаплазия $\rightarrow$ & Светлоклеточная аденокарцинома \\
\hline Серозная папиллярная метаплазия $\rightarrow$ & Серозная карцинома \\
\hline
\end{tabular}

ТАБЛИЦА 3.

ВОЗМОЖНЫЕ ТИПЫ

ЭНДОМЕТРИАЛЬНЫХ

КАРЦИНОМ, ВОЗНИКШИЕ

НА ФОНЕ ПРИМЕНЕНИЯ

ГОРМОНАЛЬНЫХ ПРЕПАРАТОВ применяющиеся для медикаментозного прерывания беременности, экстренной контрацепции и лечения лейомиомы матки. На фоне их применения были выявлены особые изменения эндометрия, не встречавшиеся ранее в клинической практике. При назначении всех модуляторов прогестероновых рецепторов в течение длительного времени наблюдалась асимметрия стромального и эпителиального компонентов с преобладанием последнего и выраженная кистозная дилатация желез с признаками одновременно эстрогенного (митотического) и прогестагенного (секреторного, апоптического) влияния. Совокупность изменений была настолько нова, что группа экспертов пришла к выводу о необходимости разработки новой терминологии. Данная гистологическая картина была охарактеризована для МПРассоциированных изменений эндометрия [28]. В то же время при анализе результатов многоцентровых исследований не выявлено случаев возникновения рака эндометрия на фоне применения препаратов данной группы, что свидетельствует о их относительной безопасности (табл. 4). Однако эта информация, несмотря

ТАБЛИЦА 4

\begin{tabular}{|c|c|c|c|c|}
\hline $\begin{array}{l}\text { Препарат, доза, дли- } \\
\text { тельность применения }\end{array}$ & $\begin{array}{c}\text { Изменения эндо- } \\
\text { метрия }\end{array}$ & Гиперплазия & $\begin{array}{c}\text { Толщина эндо- } \\
\text { метрия }\end{array}$ & $\begin{array}{c}\text { Рак } \\
\text { эндометрия }\end{array}$ \\
\hline $\begin{array}{l}\text { Мифепристон } \\
\text { (Mifepristone) } \\
5 \text { или } 10 \text { мг/сут } \\
\text { в течение } 3 \text { мес }\end{array}$ & $\begin{array}{c}\text { Типичные для } \\
\text { МПР }\end{array}$ & У $14 \%$ женщин & $\begin{array}{c}\text { Неизменна/уве- } \\
\text { личение }\end{array}$ & - \\
\hline $\begin{array}{l}\text { Преллекс (Proellex) } \\
12,5 ; 25 \text { или } 50 \text { мг/сут } \\
\text { в течение } 3 \text { мес }\end{array}$ & $\begin{array}{l}\text { Типичные для } \\
\text { МПР + атрофия }\end{array}$ & - & $\begin{array}{c}\text { Увеличивается, } \\
\text { что связано с } \\
\text { кистозной дила- } \\
\text { тацией желез, а } \\
\text { не с истинной ГЭ }\end{array}$ & - \\
\hline $\begin{array}{l}\text { Улипристал (Ulipristal) } \\
2,5 ; 5 \text { и } 10 \text { мг в течение } \\
3 \text { мес }\end{array}$ & $\begin{array}{c}\text { Типичные для } \\
\text { МПР }\end{array}$ & - & $\begin{array}{c}\text { Дозозависимо } \\
\text { снижается }\end{array}$ & - \\
\hline $\begin{array}{l}\text { Азопризнил } \\
\text { (Asoprisnil) } \\
10 \text { или } 25 \text { мг/сут в } \\
\text { течение } 3 \text { мес }\end{array}$ & $\begin{array}{c}\text { Типичные для } \\
\text { МПР + большое } \\
\text { количество } \\
\text { толстостенных } \\
\text { артериол }\end{array}$ & - & Снижается & - \\
\hline
\end{tabular}

ТАБЛИЦА 4.

ИЗМЕНЕНИЯ ЭНДОМЕТРИЯ НА ФОНЕ ПРИМЕНЕНИЯ МПР (АНАЛИЗ МНОГОЦЕНТРОВЫХ РАНДОМИЗИРОВАННЫХ ИССЛЕДОВАНИЙ III ФАЗЫ эндометрий будет способен обеспечить процесс имплантации оплодотворенной яйцеклетки и дальнейшее вынашивание беременности, а также каким образом он поведет себя в период менопаузы.

Адекватная клиническая трактовка результатов гистологического исследования эндометрия чрезвычайно важна не только с целью оценки потенциального онкориска, но и как метод опосредованного определения гормонального гомеостаза и реакции органов-мишеней.

Известно, что эстрогены стимулируют рост желез, а гестагены - стромы эндометрия. Поэтому от соотношения данных гормонов зависит состояние эндометрия, так как только оптимальное соотношение концентраций эстрадиола и прогестерона в фолликулярной и лютеиновой фразах менструального цикла обеспечивает полноценную пролиферацию и секреторную трансформацию эндометрия.

Сниженные концентрации эстрогенов и прогестерона приводят к недоразвитию как стромы, так и желез, т.е. к атрофии эндометрия.

Высокое содержание прогестерона при сниженной концентрации эстрогенов обусловливает децидуоподобную трансформацию стромы на фроне недоразвития желез, что может клинически проявляться гестагенными кровотечениями прорыва. Подобная картина может наблюдаться под воздействием гестагенов или при использовании комбинированных эстрогенгестагенных препаратов с преобладанием гестагенного компонента [20, 31].

Сочетание высоких концентраций гестагенов и эстрогенов способствует инволютивным изменениям в железах и строме или предецидуальным изменениям стромы эндометрия. Такая картина может наблюдаться при применении высокодозированных КОК, особенно в повышенных дозах по гемостатической схеме.

Высокие дозы эстрогенов на фоне сниженной продукции прогестерона приводят к чрезмерной пролиферации и кистозному расширению желез, недоразвитию стромы, что способствует преобладанию железистого компонента, т.е. возникновению ГЭ.

Таким образом, кратковременная гиперэстрогенемия влечет за собой развитие простой гиперплазии, а хроническая абсолютная или относительная гиперэстрогенемия (или гипогестагенемия) обусловливает развитие комплексной (аденоматозной) гиперплазии. Именно поэтому в лечении данных видов ГЭ с успехом применяются гестагены.

Для своевременного выявления и правильной клинической интерпретации морфологических изменений эндометрия, а также установления их гормонозависимости и адекватного дифференцированного лечения гиперпластического процесса важна этапность и применение современных высокоинформативных методик. на отсутствие сообщений о малигнизации, не может оставаться вне внимания акушеровгинекологов, так как сегодня все еще не известны дальнейшие морфологические и функциональные возможности таких изменений в эндометрии. Не совсем ясно, насколько этот 
АНАЛИТИЧЕСКИЙ ОБЗОР

В ранней доклинической диагностике гиперпластических процессов эндометрия для определения показаний к гистологическому исследованию применяется ультразвуковая диагностика, преимущественно трансвагинальная эхография. В ходе исследования оценивают толщину эндометрия, эндометриальноматочный коэффрициент (отношение толщины эндометрия к величине передне-заднего размера матки), однородность структуры, особенности эхогенности и контуров М-эхо.

Основными ультразвуковыми критериями ГЭ в репродуктивном периоде являются увеличение его толщины во II фазе менструального цикла более 16 мм и эндометриально-маточный коэффрициент более 0,33, а в постменопаузе соответственно более 4 мм и 0,15 [18]. Наличие вышеназванных сонографических признаков является показанием к проведению гистологического исследования эндометрия даже при отсутствии симптомов.

В публикациях последних лет отмечается целесообразность проведения допплерографиических и допплерометрических исследований эндометрия для количественной оценки его кровоснабжения. УЗИ проводится с расчетом объема и трехмерных допплерометрических индексов, а именно:

5 индекса васкуляризации (vascularization index, VI) - отображает насыщенность ткани сосудами, выражается в процентах;

5 индекса кровотока (flow index, FI) - показывает среднюю интенсивность кровотока, выражается целым числом от 0 до 100;

5 отношения васкуляризации к кровотоку (vascularization-flow index, VFI) - характеризует как васкуляризацию, так и кровоток и выражается целым числом от 0 до 100

Как видно из таблицы 5, в случае наличия рака эндометрия вышеперечисленные показатели существенно отличаются от таковых при доброкачественных гиперпролиферативных процессах в эндометрии. Так, показатели VI и FI при возникновении аденокарциномы эндометрия в 5-10 раз выше, чем при гиперплазии, полипах, кистозной атрофии эндометрия. Доброкачественные процессы в эндометрии характеризуются более низким значением вышеуказанных индексов. Интенсивное кровоснабжение эндометрия с множеством цветовых локусов, наличием лакун и анастомозов при цветном картировании и высокими показателями VI, FI, VFI могут указывать на малигнизацию процесса. Значение FI в случаях малигнизации субмукозной миомы также возрастает по сравнению с доброкачественными процессами. Поэтому повышение этих показателей должно насторожить клинициста и служить показанием для проведения гистероскопии и гистологического исследования $[12,19]$.

\begin{tabular}{|c|c|c|c|c|c|}
\hline & ТАБЛИЦА 5 \\
\hline $\begin{array}{c}\text { Результат } \\
\text { пульсогемоинди- } \\
\text { кации }\end{array}$ & $\begin{array}{c}\text { Толщина } \\
\text { эндометрия, } \\
\text { мм }\end{array}$ & $\begin{array}{c}\text { Объем эндо- } \\
\text { метрия, мл }\end{array}$ & VI, \% & $\mathrm{FI}$ & VFI \\
\hline Аденокарцинома & $\begin{array}{c}16,34 \\
(13,69-18,98)\end{array}$ & $\begin{array}{c}8,70 \\
(6,73-10,67)\end{array}$ & $\begin{array}{c}18,97 \\
(13,61-24,33)\end{array}$ & $\begin{array}{c}29,43 \\
(27,19-31,67)\end{array}$ & $\begin{array}{c}6,00 \\
(3,98-8,02)\end{array}$ \\
\hline Гиперплазия & $\begin{array}{c}9,21 \\
(6,71-11,71)\end{array}$ & $\begin{array}{c}3,19 \\
(1,38-5,00)\end{array}$ & $\begin{array}{c}1,30 \\
(0,15-2,76)\end{array}$ & $\begin{array}{c}17,81 \\
(11,07-24,55)\end{array}$ & $\begin{array}{c}0,43 \\
(0,02-0,89)\end{array}$ \\
\hline Полип & $\begin{array}{c}11,38 \\
(9,34-13,42)\end{array}$ & $\begin{array}{c}3,38 \\
(1,61-5,16)\end{array}$ & $\begin{array}{c}3,11 \\
(1,21-5,01)\end{array}$ & $\begin{array}{c}21,89 \\
(16,60-27,19)\end{array}$ & $\begin{array}{c}0,92 \\
(0,33-1,51)\end{array}$ \\
\hline $\begin{array}{l}\text { Кистозная } \\
\text { атрофрия }\end{array}$ & $\begin{array}{c}9,41 \\
(6,48-12,33)\end{array}$ & $\begin{array}{c}2,55 \\
(0,74-4,36)\end{array}$ & $\begin{array}{c}2,36 \\
(0,49-5,22)\end{array}$ & $\begin{array}{c}25,43 \\
(21,20-29,68)\end{array}$ & $\begin{array}{c}0,68 \\
(0,20-1,56)\end{array}$ \\
\hline $\begin{array}{l}\text { Субмукозная } \\
\text { миома }\end{array}$ & $\begin{array}{c}9,20 \\
(3,15-15,24)\end{array}$ & $\begin{array}{c}2,43 \\
(0,38-5,25)\end{array}$ & $\begin{array}{c}3,50 \\
(0,42-7,42)\end{array}$ & $\begin{array}{c}26,72 \\
(16,57-36,86)\end{array}$ & $\begin{array}{c}1,08 \\
(0,47-2,62)\end{array}$ \\
\hline
\end{tabular}

Важный этап диагностики ГЭ - забор материала, поскольку окончательным является гистологический диагноз. Еще в 1843 г. Rekamier изобрел кюретку, и с тех пор этот инструмент используется для получения образцов эндометрия. Основным методом получения материала на протяжении более века является лечебно-диагностическое раздельное выскабливание цервикального канала и полости матки. Однако в связи с травматичностью и возможными осложнениями, связанными с проведением кюретажа и анестезии, остается актуальным вопрос использования более щадящих методов получения эндометрия.

В 1954 г. McBride впервые предложил использовать биопсию эндометрия, однако полученные при этом образцы ткани только в 13\% случаев соответствовали материалу, удаленному при кюретаже матки. В 1970 г. в клиническую практику был внедрен метод аспирационной биопсии с использованием вакуумной эндоретты (кюретки) диаметром 3 мм, позволяющий в амбулаторных условиях получить образцы эндометрия без использования анестезии. Однако рядом исследований, проведенных в 90-е годы, в 18-42\% случаев выявлено гистологическое несоответствие образцов эндометрия, полученных при биопсии и кюретаже [18, 19, 25, 30]. Таким образом, вакуумная биопсия эндометрия с применением эндоретты под контролем УЗИ может использоваться только как метод мониторинга состояния эндометрия у женщин, применяющих гормонотерапию [18, 19, 25, 30].

Тем не менее даже полное раздельное диагностическое выскабливание цервикального канала и полости матки не обеспечивает стопроцентную надежность в адекватном получении материала [31]. Подтверждением тому стало проведенное в 2009 г. исследование, в котором было доказано, что при аспирационной биопсии неполная диагностика рака имела место в 45\% случаев, в то время как при раздельном диагностическом выскабливании - в 30\%. Другими словами, практически более трети случаев рака эндометрия были пропущены при проведении полного кюретажа цервикального канала и полости матки (табл. 6) [31]. ДОППЛЕРОМЕТРИЧЕСКИЕ ПОКАЗАТЕЛИ ПРИ РАЗЛИЧНЫХ ВАРИАНТАХ ГИПЕРПЛАСТИЧЕСКИХ ПРОЦЕССОВ

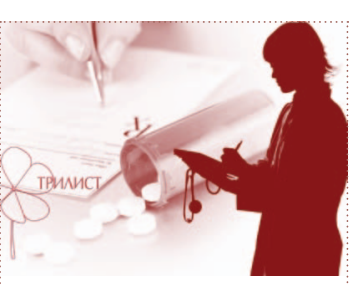

Кратковременная гиперэстрогенемия влечет за собой развитие простой гиперплазии, а хроническая абсолютная или относительная гиперэстрогенемия (или гипогестагенемия) комплексной (аденоматозной) гиперплазии. Поэтому

в лечении этих видов ГЭ высока эффективность гестагенов 
ТАБЛИЦА 6

\begin{tabular}{|l|c|c|c|}
\hline \multicolumn{1}{|c|}{ Вид патологии } & $\begin{array}{c}\text { Раздельное диагно- } \\
\text { стическое выскабли- } \\
\text { вание, \% }\end{array}$ & $\begin{array}{c}\text { Аспирационная биоп- } \\
\text { сия эндометрия, \% }\end{array}$ & Величина p \\
\hline Недиагностированный рак & 30 & 45 & $\mathrm{p}<0,001$ \\
\hline Инвазия в эндометрий & 18 & 25 & $\mathrm{p}=0,05$ \\
\hline
\end{tabular}

ТАБЛИЦА 6.

СРАВНИТЕЛЬНАЯ

ХАРАКТЕРИСТИКА

РАЗДЕЛЬНОГО

ДИАГНОСТИЧЕСКОГО

ВЫСКАБЛИВАНИЯ И БИОПСИИ

ЭНДОМЕТРИЯ ПРИ НЕПОЛНОМ

УСТАНОВЛЕНИИ ДИАГНОЗА

РАКА ЭНДОМЕТРИЯ

И ИНВАЗИВНОЙ КАРЦИНОМЫ

В этом контексте особую значимость приобретают способы визуализации эндометрия, диагностическая ценность которых значительно повысилась с развитием современных оптических систем. Гистероскопия позволяет визуализировать патологические изменения эндометрия и определять их особенности и локализацию, контролировать качество диагностического выскабливания с прицельным удалением возможных остатков гиперплазированного эндометрия или полипов при минимальном травматизме здоровой ткани и выполнять внутриматочные операции с применением электро- и лазерной хирургии.

Преимуществом гистероскопии является возможность ее использования в амбулаторных условиях (наружный диаметр инструментов для офисной гистероскопии составляет 2,5-3 мм) и возможность выполнения эндоскопических операций. Панорамная гистероскопия обеспечивает прямой осмотр всей полости матки и способствует установлению топографической связи выявленных аномалий в отношении всего эндометрия, что помогает диффреренцировать субмукозные фибромиомы, полипы и региональные утолщения эндометрия, оценить сосудистый рисунок эндометрия. Использование двойного канала обеспечивает проведение хирургических манипуляций. Сегодня доказаны преимущества гистероскопии в удалении полипов эндометрия (уровень доказательности В).

Учитывая, что неатипические виды ГЭ являются результатом гиперэстрогении, ключевым моментом в лечении данной патологии является именно коррекция преобладания эстрогенов, т.е. гормонотерапия [8, 15, 18].

Согласно протоколу, утвержденному приказом МЗ Украины от 31.12.2004 г. № 676, лечение ГЭ включает следующие этапы:

І этап - удаление измененного эндометрия с последующим морфологическим исследованием. Детализация дальнейшей тактики лечения проводится в зависимости от вида патологии эндометрия.

II этап - гормональная терапия, направленная на супрессию эндометрия с применением гестагенов и/или аГнРГ. Длительность терапии - 6 мес с повторным гистологическим исследованием каждые 3 мес (в случае простой ГЭ возможна гистология через 6 мес при отсутствии ультразвуковых критериев гиперплазии). При наличии гиперплазии через 3 мес терапии проводится ее коррекция, а при атипической гиперплазии показана консультация онколога-гинеколога.
III этап - оптимизация гормонального статуса с целью предупреждения дальнейшего развития гиперэстрогенемии.

Женщинам репродуктивного возраста показано:

восстановление двухфазного менструального цикла при необходимости сохранения репродуктивной функции;

применение KOK с гестагеном, обладающим антипролиферативным действием на эндометрий;

локальная гестагенная гормонотерапия (левоноргестрелвысвобождающая внутриматочная система).

В климактерическом периоде проводится меностаз, при необходимости - с использованием аГнРГ, а при показаниях к ЗГТ применяют монофазные препараты, содержащие гестаген с выраженным антипролиферативным эффектом, либо проводится ЗГТ на фоне локальной гормонотерапии.

IV этап - диспансерное наблюдение на протяжении 5 лет после эффрективной гормональной терапии и в течение 6 мес после оперативного лечения (УЗИ органов малого таза выполняют два раза в год).

На протяжении длительного периода времени для лечения ГЭ с успехом применяют гестагены. Их действие направлено на:

э подавление митотической активности эндои миометрия, что приводит к увеличению продукции эстрадиол-конвертирующих энзимов;

у угнетение инкорпорации тимидина клетками эндо- и миометрия и снижение их чувствительности к эстрогенам;

с снижение синтеза матричных металлопротеиназ - ферментов, обеспечивающих процессы ремоделирования в эндометрии;

5 угнетение активности инсулиноподобного фрактора роста 1 путем повышения продукции связывающего его протеина;

п повышение активности тромбоспондина 1 - экстраклеточного медиатора, угнетающего ангиогенез в эндометрии;

у угнетение эстроген-медиированной транскрипции онкогенов [32, 33].

Вышеперечисленные механизмы действия гестагенов способствуют железистой супрессии эндометрия.

Режимы и схемы применения различных прогестагенов определяются биологическими эфрфектами разных доз препаратов, чувствительностью к гестагенам эпителия и стромы эндометрия в различные фразы менструального цикла и, соответственно, целью, которую ставит перед собой клиницист, назначая лечение того или иного патологического состояния.

Следует помнить, что риск развития карциномы эндометрия значительно повышается при наличии ожирения, метаболического синдрома, хронических заболеваний печени, 
рецидивирующих дисфункциональных маточных кровотечений, бесплодия, хронической ановуляции, синдрома поликистозных яичников, эстроген-продуцирующих опухолей яичников, поздней менопаузы, длительного применения тамоксифена, семейного варианта неоплазии молочной железы, яичников, толстого кишечника. Поэтому для лечения ГЭ желательно выбирать метаболически нейтральный гестаген, учитывая обменно-эндокринные риски малигнизации гиперпластических процессов, а также дополнительные положительные эффректы гестагена $[23,27]$.

Нередко гиперпролиферативные процессы матки, в т.ч. эндометрия, возникают на фоне хронического воспаления. Повышенная продукция фракторов роста иммунокомпетентными клетками в очаге воспаления стимулирует митотическую активность клеток и развитие гиперпластических процессов. Ранее проведенные нами исследования [18, 19] показали, что на фроне хронического эндометрита изменяется экспрессия гестагенных рецепторов, которые становятся нечувствительными к воздействию прогестерона и его аналогов. Эти свойства, играя немаловажную роль в развитии гиперпластических процессов, очевидно, нередко служат и причиной неэффрективности гестагенной терапии. Поэтому в комплексе лечения гиперпролиферативных процессов эндометрия на фроне хронического эндометрита обязательно должна входить этиотропная противовоспалительная терапия $[1,14,19,22]$.

Учитывая приведенные выше результаты последних исследований о том, что длительное применение гестагенов может стимулировать развитие муцинозных и светлоклеточных аденокарцином, в клинической практике с целью лечения комплексных ГЭ все шире применяются аГнРГ. Основное действие этих препаратов основано на создании медикаментозной менопаузы путем снижения продукции эстрогенов и прогестерона в яичниках за счет десенситизации рецепторов гипофиза и снижения его гонадотропной функции. Уменьшение продукции эстрогенов приводит к недоразвитию желез эндометрия, гестагенов - желез стромы, а также к снижению содержания жидкости в клетках и межклеточных пространствах и соответственно к атрофии эндометрия. Однако механизмы действия аГнРГ не ограничиваются блокадой стероидогенеза в яичниках. На сегодняшний день доказано, что препараты этой группы угнетают активность некоторых факторов роста (в частности эпидермального фактора роста), снижают экспрессию рецепторов к эпидермальному и трансорормирующему фракторам роста; повышают экспрессию генов Fas и Fast; активизируют апоптоз; тормозят захват тимидина миоцитами, уменьшая пролиферацию; угнетают ароматазную систему, способствуя уменьшению локальной продукции эстрогенов, а также снижают чувствительность к эстрогенам [18, 21].

Показания к применению аГнРГ при лечении ГЭ:

3 простая неатипическая ГЭ в пери- и постменопаузе;

з рецидивирующее течение простой неатипической ГЭ в репродуктивном возрасте;

з комплексная неатипическая ГЭ в репродуктивном возрасте и в перименопаузе;

(9 рецидивирующая неатипическая комплексная ГЭ в репродуктивном периоде;

атипическая простая и комплексная ГЭ в репродуктивном возрасте;

б ГЭ в сочетании с миомой матки и/или аденомиозом.
Недостатком медикаментозной менопаузы является возможность возникновения побочных эффректов, обусловленных дефицитом эстрогенов, что несколько ограничивает их применение. В исследованиях было выявлено, что низкий уровень эстрадиола (ниже 30 нг/мг) снижает прогрессию эстрогензависимых процессов, но приводит к потере костной массы и выраженным вегетативным проявлениям. Умеренно высокий уровень эстрадиола необходим для предупреждения остеопороза, однако вызывает резкое прогрессирование гиперпролиферативных процессов. Целевой уровень эстрадиола - около 30 пг/мл, что оптимально для эффрективного лечения и менее опасно с точки зрении вероятности потери костной массы. Эффективность применяющихся в современной клинической практике аГнРГ в отношении ГЭ, вероятно, обеспечивается синергичным воздействием гипоэстрогенного влияния и описанных выше дополнительных механизмов действия. При этом исследования показали, что назначение такого препарата, как диферелин (действующее вещество трипторелин), сопровождается снижением концентрации эстрадиола в сыворотке крови именно до 30 пг/мл, что обеспечивает и клинический эффект, и минимизацию побочных действий $[11,20]$.

В случаях неэффрективности консервативной терапии и рецидивирования ГЭ показано оперативное лечение. При неатипических формах ГЭ, особенно у женщин репродуктивного возраста, в последние годы все чаще используется гистероскопическая резекция или абляция эндометрия, а при атипических фрормах операцией первого выбора является экстирпация матки.

Показаниями к оперативному лечению больных с гиперпролиферативными процессами эндометрия являются:

в репродуктивном периоде:

- атипическая комплексная ГЭ - при отсутствии эфректа от консервативной терапии в течение 3 мес;

- простая атипическая и комплексная неатипическая ГЭ - при неэффективности консервативной терапии в течение 6 мес;

в климактерическом периоде:

- комплексная атипическая гиперплазия - при установлении диагноза;

- простая атипическая и комплексная неатипическая гиперплазии - при отсутствии эфффекта от консервативной терапии в течение 3 мес.

Следует акцентировать внимание на нецелесообразности необоснованного расширения показаний к гистерэктомии при ГЭ без предварительной консервативной терапии, особенно у пациенток в возрасте до 45 лет. В то же время важно подчеркнуть возможность расширения вышеупомянутых показаний к оперативному лечению, определяя их индивидуально в каждом конкретном случае с учетом степени риска возможной малигнизации. Сегодня имеются возможности органосохраняющего хирургического лечения ГЭ - резекция эндометрия с применением электрохирургической и радиоволновой энергии.

Учитывая омоложение данной патологии и откладывание многими женщинами на более зрелый возраст реализации репродуктивной функции, все более важными становятся исследования по изучению патогенетических звеньев ГЭ и рисков ее малигнизации, что потенциально расширяет возможности органосохраняющей терапии. 


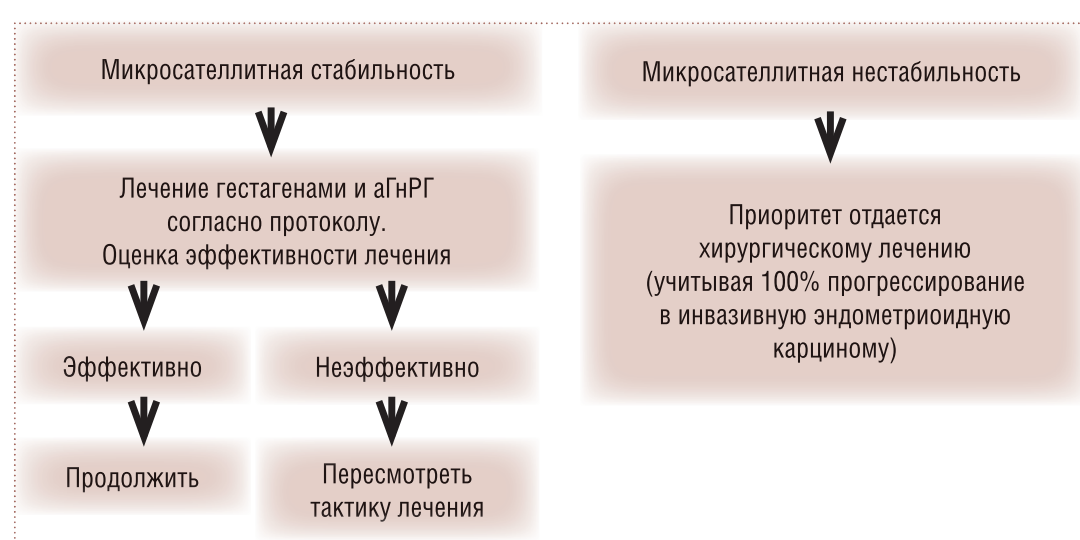

CXEMA 2.

ПЕРСПЕКТИВА ОПРЕДЕЛЕНИЯ

ТАКТИКИ ЛЕЧЕНИЯ ГЭ

В ЗАВИСИМОСТИ ОТ

ГЕНЕТИЧЕСКОГО ОБСЛЕДОВАНИЯ
В этой связи огромный интерес представляют исследования, направленные на выявление генетической детерминированности ГЭ и вероятности их малигнизации.

На кафедре акушерства и гинекологии Одесского национального медицинского университета проведены генетические исследования, в ходе которых был выявлен 31 сайт фрагильности хромосом: 4 сайта фррагильности (1q2.5-4.1; 2q2.4-31; 4q1.5; 5g1.4) у пациенток с атипической ГЭ и аденокарциномой эндометрия как на фоне отягощенного семейного анамнеза, так и без него; 4 фррагильных сайта (17.q2.2; 1q2.2; 1q1.4; 2p1.5-2.2) - у больных с отягощенной онкологической наследственностью, не встречающиеся у женщин без отягощенного семейного анамнеза. На основании этого был сделан вывод о генетической детерминированности и полигенной природе опухолевой патологии эндометрия [5, 6]. Проведенные молекулярногенетические исследования позволили обнаружить изменения в группах онкогенов, геновсупрессоров, генов стероидных рецепторов и стероидогенеза, а также генов детоксикации при наличии рака эндометрия. Обоснованна целесообразность проведения на доклиническом этапе генетического скрининга с применением ДНК-чиповой технологии, объективизирующей формирование групп риска возникновения опухолей эндометрия, в которых при развитии упомянутой патологии важно своевременно применять органосохраняющие малоинвазивные хирургические технологии ввиду высокого риска ее малигнизации [5].

Полученные нами результаты соответствуют данным исследований Gisella Dallenbach-Hellweg et al. (2010), Francesco Colotta et al. (2009), в которых доказано, что у женщин младше 40 лет каждая третья комплексная или атипическая гиперплазия является результатом микросателлитной нестабильности ${ }^{1}$, и это 100-процентный шанс прогрессирования в инвазивную эндометриоидную карциному в отличие от микросателлитностабильных гиперплазий, которые имеют низкий уровень прогрессии и высокий уровень регрес- сии на фроне эндо- или экзогенной прогестероновой стимуляции (табл. 6) [26]. В настоящее время эти данные еще не получили широкого практического применения, однако ввиду наличия тесной связи перспектив лечения и возможности малигнизации ГЭ, возможно, с широким внедрением методов определения генетической нестабильности в практику, тактика лечения будет зависеть от результатов упомянутого обследования (схема 2). При стабильном геноме перспективной является консервативная терапия, в то время как в случаях его микросателлитной нестабильности целесообразно склоняться в сторону хирургического лечения, учитывая высокий риск прогрессирования процесса с переходом в инвазивную карциному [5, 6, 23, 26].

Кроме того, выявлено, что снижение активности NO-синтетазы как универсального маркера дисрегуляции, происходящее синхронно с уменьшением количества рецепторов к эстрадиолу и прогестерону в сторону их исчезновения при прогрессировании заболевания, может быть прогностическим критерием перехода гиперпластических процессов в неопластические [5, 12].

Таким образом, возможности современной диагностики и терапии гиперпластических процессов эндометрия постоянно совершенствуются, открывая все новые перспективы их лечения. Обобщая вышеизложенное, можно сделать вывод, что залогом успеха терапии гиперпролиферативных процессов эндометрия является адекватная трактовка результатов морфологического исследования и понимание этиологии, патологенеза и генетической предрасположенности выявленных изменений. Этому способствует своевременная качественная диагностика с использованием трансвагинальной сонографии, допплерометрии, гистероскопии, а также применение унифицированных современных классификаций ГЭ. Возможность развития ятрогенных изменений эндометрия диктует необходимость взвешенного подхода и осторожного назначения любых гормональных препаратов. С внедрением в клиническую практику методики создания медикаментозной менопаузы при помощи аГнРГ, а также миниинвазивных эндоскопических хирургических методик существенно расширились возможности эфрфективного органосохраняющего лечения сложных видов гиперплазий. Вместе с тем значительно сузились показания к радикальной гистерэктомии, что особенно актуально при лечении женщин детородного возраста с нереализованной репродуктивной функцией, особенно в условиях сложной демографической ситуации в Украине.

Список литературы в количестве 33 источников представлен на сайте www.reproduct-endo.com.ua

${ }^{1}$ Микросателлитная нестабильность - индикатор мутаторного феенотипа и диагностический признак десекта пострепликативной репарации, что используется для деления опухолей и линий опухолевых клеток на RER+ и RER- (RER - аббревиатура слов replication errors). Микросателлитная нестабильность как результат нарушения метаболизма ДНК, ее репликации и репарации является причиной развития опухолей (прим. ред.). 